\title{
Sijunzi Tang, Codonopsis Pilosula Polysaccharide and Saccharomyces Boulardii Modulate the Gut Microbiome to Alleviate Dss-Induced Colitis in Mice
}

\author{
Xinjun Chen ${ }^{1}$, Anping Li ${ }^{5,6}$, Pingrong Yang ${ }^{5,6}$, Juan Wen ${ }^{2,3,4}$, Yaping Jing ${ }^{2,3,4}$, Chenhui $\mathrm{Li}^{2,3,4}$, Junshu Wei ${ }^{2,3,4}$, \\ Tuanjie $\mathrm{Che}^{3}$, Xiao Ma ${ }^{5,6}$, Pingshun Song ${ }^{5,6}$ and Chunjiang Zhang ${ }^{2,3,4 *}$ \\ ${ }^{1}$ Laboratory of Pathogenic Biology and Immunology, China \\ ${ }^{2}$ School of Life Sciences, China \\ ${ }^{3}$ Gansu Key Laboratory of Functional Genomics and Molecular Diagnosis, China
}

${ }^{4}$ Gansu Key Laboratory of Biomonitoring and Bioremediation for Environmental Pollution, China

${ }^{5}$ Gansu Institute of Drug Control, China

${ }^{6}$ Gansu Tebetan Herbal Medicine Engineering Laboratory of Testing and Detection, China

*Corresponding author: Chunjiang Zhang, School of Life Sciences, Gansu Key

Laboratory of Functional Genomics and Molecular Diagnosis, Lanzhou, China.

Received Date: December 07, 2018

Published Date: December 20, 2018

\begin{abstract}
Inflammatory bowel disease (IBD) is usually considered to be more frequent in Western countries, but now a recent increase in the incidence and prevalence has been observed in China. Chinese herbal medicine as the main example of complementary and alternative medicine (CAM) is widely used by Chinese patients. However, the underlying mechanism of herbal medicine in alleviating IBD is still covered.

The gut microbiome plays a key role in which has spurred the development of novel therapeutics aimed at restoring microbial community structure. So, probiotic represents another widely used CAM in treating IBD. Saccharomyces boulardii has showed great beneficial effect on diarrhea, but it is rarely used in IBD.

In this study, we aim to evaluate the efficacy and elucidate the mechanisms of four potential CAM drugs named S. boulardii, SJZT, Dangshen [Codonopsis pilosula (French.) Nannf] polysaccharide or S. boulardii in combination with Dangshen polysaccharide during treatment with dextran sulphate sodium (DSS)-induced mice colitis. The gut microbiome of mice before and post colitis treatments were performed by $16 \mathrm{~S}$ rRNA-based microbiome analysis. All four drugs profoundly inhibited weight loss, colon shortening, and ameliorated histological damage as well as DSS-induced dysbiosis in mice. Beneficial bacteria- especially short chain fatty acid (SCFA)-producing genera were remarkably enriched in the treated mice. Roseburia, Anaerovorax and Lactobacillus were selectively enriched by S. boulardii, while Butyrivibrio, Quinella and Anaerotruncus were selectively enriched by SJZT. Most notably, Dangshen polysaccharide selectively enriched Bifidobacterium, Arthrobacter, Akkermansia, Anaerovorax, Roseburia, Prevotella, Dialister, Megamonas, Faecalibacterium and Subdoligranulum. S. boulardii, Sijunzi decoction, and its main component, Dangshen polysaccharide, are effective in alleviating DSS-induced colitis. Furthermore, Dangshen polysaccharide is a powerful prebiotic that selectively promotes probiotic growth, especially SCFA-producing bacteria. These findings suggest that modulating the gut microbiome may be the common mechanism whereby these drugs improve colonic health.
\end{abstract}

\section{Introduction}

Inflammatory bowel disease (IBD) is the term used to describe chronic intestinal inflammatory conditions, including ulcerative colitis (UC) and Crohn's disease (CD). While IBD is especially common among western populations, the incidence of IBD in China has increased rapidly in recent years because of the widely adopted westernized lifestyle [1]. The etiology and pathogenesis of IBD are not fully understood, and effective therapeutics are lacking.

A healthy, balanced intestinal microbiome plays a key role in maintaining whole-body homeostasis. Significant alterations to the gut microbiome, referred to as dysbiosis, are present in most 
chronic inflammatory disease. Intestinal dysbiosis is a defining feature of IBD, with some even considering it to be causative. Indeed, manipulating the gut microbiome has been shown to greatly alleviate the symptoms of IBD [2]. Pro- and prebiotics are two widely adopted therapeutic strategies that are also great examples of complementary and alternative medicines (CAMs) being used in clinic.

Traditional Chinese medicines have also shown remarkable curative effects as CAMs in IBD patients. Sijunzi Tang (SJZT) decoction is a classical herbal formula composed of Dangshen [Codonopsis pilosula (Franch.) Nannf], Baizu (Atractylodes macrocephalae Koidz), Fuling [Poria $\operatorname{cocos}$ (Schw). Wolf] and Gancao (Glycyrrhiza uralensis Fisch). Related researches claimed that SJZT were efficiently in treating UC patients in China [3]. Its mechanism of action however remains unknown. Recent study suggests that Chinese herbal medicines could potentially be used to modulate the intestinal microbiome. Treatment with Gegen Qinlian decoction for example promotes the growth of beneficial bacteria, especially Faecali bacterium prausnitzii, to alleviate type II diabetes [3]. We therefore hypothesized that SJZT decoction could also relieve IBD symptoms by altering the gut microbiome.

Furthermore, based on the theory of Chinese medicine, watersoluble polysaccharides are the key compounds in decoctions. Dangshen polysaccharide, a macromolecular substance that is hard to digest, consists of monosaccharides like D-galactose, D-rhamnose and $\mathrm{D}$-arabinose and their derivatives, a backbone of $(1 \rightarrow 3)$-linked$\beta$-D-galactopyranosyl, $(1 \rightarrow 2,3)$-linked- $\beta$-Dgalactopyranosyl and $(1 \rightarrow 3)$-linked- $\alpha$-D-rhamnopyranosyl residues [4]. Based on the fact that herbal polysaccharides such as Lentinula edodes [5] and Ganoderma lucid me [6] are very effective at modulating the gut microbiome, we hypothesized that non-digestible Dangshen polysaccharide might favor the growth of specific, beneficial microbes, i.e. act as a prebiotic.

The fungi Saccharomyces boulardii is a new probiotic that is resistant to antibiotics and low $\mathrm{pH}$ and grows very well at $37{ }^{\circ} \mathrm{C}$ [7]. Although widely used in preventing and treating diarrhea, it is rarely used to treat IBD. Most studies on S. boulardii in animal colitis models focus mainly on immune-related mechanisms [8,9]. Recently administration of S. boulardii has been reported to exert its therapeutic effects by altering the gut microbiome in obese and type 2 diabetic mice [10]. Whether S. boulardii can change microbial community structure to relieve IBD symptoms remains to be seen.

The aim of this study was to determine the effect of Sijunzi decoction, Dangshen polysaccharides and Saccharomyces boulardii on microbial diversity and composition during the treatment of DSS-induced acute colitis in mice, using high-throughput 16S-based sequencing.

\section{Material and Methods}

\section{S. boulardii, Sijunzi decoction and dangshen polysaccharide preparation}

S. boulardii sachets were purchased from Laboratoires BIOCODEX (Bioflor ${ }^{\circledR}$, France). In order to remove any contaminants a pure S. boulardii culture was obtained by inoculating the lyophilized yeast in YPD broth with shaking at $37^{\circ} \mathrm{C}$ for 18 hours; $\mathrm{S}$. boulardii was then harvested and concentrated tenfold to $109 \mathrm{CFU} /$ $\mathrm{ml}$. The yeast was centrifuged and re-suspended in PBS solution for oral administration.

The four dried raw materials used to prepare the SJZT decoction (Dangshen, Baizu, Fuling, and Gancao) were all purchased from Xukang pharmaceutical co., Itd (Lanzhou, China). The quality was all met the requirement of Chinese Pharmacopoeia (2015 Version). The voucher specimens' number were DS-20150721, BZ20150701, FL-20150718, GC-20150726 respectively. All specimens were identified by senior engineer Pin Grong Yang and deposited in the Herbarium of Chinese patent medicine test laboratory of Gansu Institute of Drug Control, Lanzhou, China.

Four materials mixed in a ratio of 9:9:9:6 and herbs were decocted twice with boiling water (10 times of the material's weight) for $60 \mathrm{~min}$ then filtered, pooled and concentrated to $1 \mathrm{~g} /$ $\mathrm{ml}$. Mice were orally administered $10 \mathrm{ml} / \mathrm{kg}$ (convert from clinical dose). Quality control of the SJZT decoction was performed by high performance liquid chromatography (HPLC) analysis of glycyrrhizin acid and liquidity from Guanaco, according to an established method [11]. Briefly, chromatographic separation was performed on a Zobax SB-C18 column $(4.6 \mathrm{~mm} \times 150 \mathrm{~mm}, 5 \mu \mathrm{m})$; the mobile phase consisted of methyl alcohol $-0.5 \%$ acetic acid; the flow rate was $1 \mathrm{ml} / \mathrm{min}$; the column temperature was $30^{\circ} \mathrm{C}$; liquidity was detected at $276 \mathrm{~nm}$, and glycyrrhizin acid at $250 \mathrm{~nm}$.

The crude polysaccharide extract was obtained from Dangshen by water decoction and alcohol precipitation. Total carbohydrate content was then determined by phenol-sulfuric acid assay based on a standard curve determined from D-glucose at six different concentrations $(\mu \mathrm{g} / \mathrm{ml})$.

\section{Animal experiments and treatment design}

The present animal study was conducted according to protocols approved by the Ethics Committee of Animal Experiments of Lanzhou University. Female C57BL/6 mice ( $n=68,16-18$ g) were purchased from the Gansu university of Chinese medicine. After acclimatization for one week, mice were housed individually in plastic cages at constant temperature $\left(21 \pm 2^{\circ} \mathrm{C}\right)$, with an alternating $12 \mathrm{~h}$ light/dark cycle, and animal chow and water were provided ad libitum. Mice were divided into six groups and treatments lasted for 30 days.

A: Dangshen polysaccharide (dissolved in PBS solution, 300 $\mathrm{mg} / \mathrm{kg}$ )

B: Saccharomyces boulardii (resuspended in PBS to a concentration $109 \mathrm{CFU} / \mathrm{ml}$ )

C: Dangshen + S. boulardii, (Dangshen polysaccharide dissolved in an S. boulardii suspension)

D: Sijunzi (SJZT decotion $(1 \mathrm{~g} / \mathrm{ml})$ at $10 \mathrm{ml} / \mathrm{kg}$; dose selection was based on that used in clinic patients)

E: Colitis (DSS, PBS solution) 
F: Normal (no DSS, PBS solution)

After 21 days of treatment, acute colitis was induced by administering 2.5\% DSS solution to groups A-E for 7 days, followed by distilled water for two days. All drugs (groups A-D) were administered throughout the study (see experimental design, Figure
1). Fresh fecal samples were collected immediately upon defecation on days 1(blank) and 21(before colitis induction), placed in sterile centrifuge tubes and stored at $-80^{\circ} \mathrm{C}$. On day 30 (post-colitis) mice were sacrificed and intestinal fecal samples were collected from the colon.

\section{$2.5 \%$ DSS}

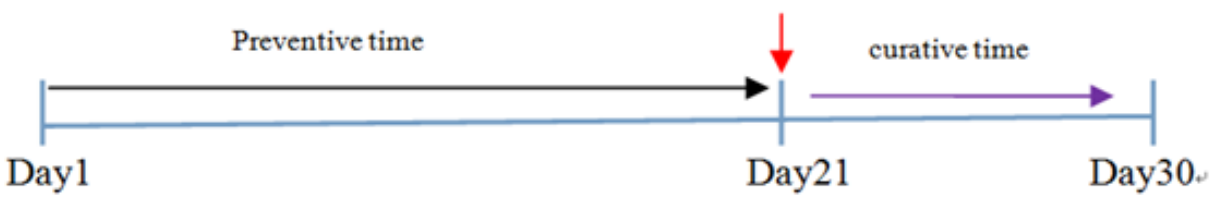

Experiment Treatment Groups:

A:Dangshen (prebiotic):

B:S.boulardii (probiotic):

C:Dangshen+ S.boulardii (synbiotic): Received probiotic suspension plus polysaccharide $(n=12)$

D:Sijunzi decoction (herbal medicine): Received $1 \mathrm{~g} / \mathrm{ml}, 10 \mathrm{ml} / \mathrm{kg}$ decoction $(\mathrm{n}=12)$

E:DSS (colitis only):

F:Normal(no colitis):
Received 300mg/kg Dangshen polysaccharide ( $\mathrm{n}=12)$

Received $10^{\wedge} 9 \mathrm{CFU} / \mathrm{ml}$ probiotic suspension $(\mathrm{n}=12)$

Received PBS solution ( $\mathrm{n}=12$ )

Received PBS solution $(\mathrm{n}=8)$

Figure 1: Outline of experimental design. DSS solution was administered for 7 days to induce acute colitis.

To evaluate the therapeutic effects of the four different treatments, mice body weight was monitored daily, and following a final assessment of colon length, inflamed colon tissues were treated with formalin and stained with hematoxylin and eosin. Inflammation was graded according to an established system [12].

\section{DNA isolation and Illumina pyrosequencing of the V3 16S rRNA gene region}

Microbial DNA was extracted from fecal samples using the Qian Gen@Stool DNA kit (Beijing, China) according to the manufacturer's instructions. DNA concentration and purity were quantified on a Nanodrop 1000 spectrophotometer (Thermo Scientific) and DNA integrity was confirmed by agarose gel electrophoresis.

Universal primers were selected to span theV3-V4hypervariable region of the 16S rRNA gene: 338F (5'-ACTCCTACGGGAGGCAGCA-3') and 806R (5'-GGACTACHVGGGTWTCTAAT-3'). In brief, PCRs were performed in $20 \mu \mathrm{l}$ reactions containing $5 \mathrm{x}$ FastPfu Buffer $(4 \mu \mathrm{l}), 10$ ng template DNA, $2 \mu \mathrm{L}$ dNTPs, $0.8 \mu \mathrm{l}$ forward and reverse primer ( $5 \mu \mathrm{l}$ ), $0.4 \mu \mathrm{l}$ Fast Pfu Polymerase and ddH2O to $20 \mu \mathrm{l}$. The PCR parameters were: $95^{\circ} \mathrm{C}$ for $3 \mathrm{~min}, 27$ cycles of $95^{\circ} \mathrm{C}$ for 30 seconds, $55^{\circ} \mathrm{C}$ for 30 seconds, $72^{\circ} \mathrm{C}$ for 45 seconds, followed by a final extension of $72^{\circ} \mathrm{C}$ for $10 \mathrm{~min}$.

PCR products were detected on a $2 \%$ agarose gel and quantified by Quanti Fluor ${ }^{\mathrm{TM}}$-ST and all samples were pooled at mean concentrations. Library preparation and pyrosequencing were performed on an Illumina MiSeq PE 300 platform by Shanghai Majorbio Technology Company.

\section{Bioinformatic and statistical analyses}

High quality sequences were assigned to each sample (demultiplexed) and clustered as operational taxonomic units (OTUs) at a threshold of $\geq 97 \%$ similarity. OTU abundance data was used to calculate alpha diversity (Shannon index), richness and rarefaction estimates using Mothur [13]. Community structure was assessed using Uni Frac principal coordinate analysis (PCoA), nonmetric multidimensional scaling (NMDS) based on Bray-Curtis distance, and hierarchical clustering, using $\mathrm{R}$ packages vegan. Heatmaps were created using Mothur and R package heatmap [14] to visualize treatment-specific changes in the microbiome.

All results are presented as the mean $( \pm$ SE). Group differences were assessed by ANOVA and the Mann-Whitney test in SPSS19.0. P-values $<0.05$ were considered significant.

\section{Results}

\section{Preparation of probiotic, Sijunzi decoction, Dangshen polysaccharide}

An S. boulardii culture was prepared from a commercial product and typical morphologic characteristics of yeast was observed (Figure 2A). Dangshen polysaccharide constituted 38.7\% of the crude, brown Dangshen polysaccharide powder (Figure 2B). HPLCbased quality control of the Sijunzi decoction was performed using glycyrrhizin acid and liquidity as indicator components (Figure 2C, 2D). The content of glycyrrhizin acid in SJZT $(1 \mathrm{~g} / \mathrm{ml})$ was $13.5 \mu \mathrm{g} /$ $\mathrm{ml}$ while that of liquidity was $5.8 \mu \mathrm{g} / \mathrm{ml}$. 
A

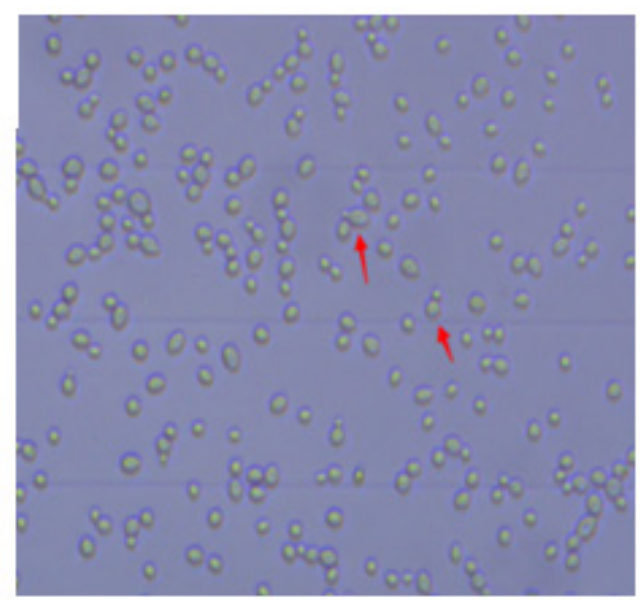

$\mathrm{C}$

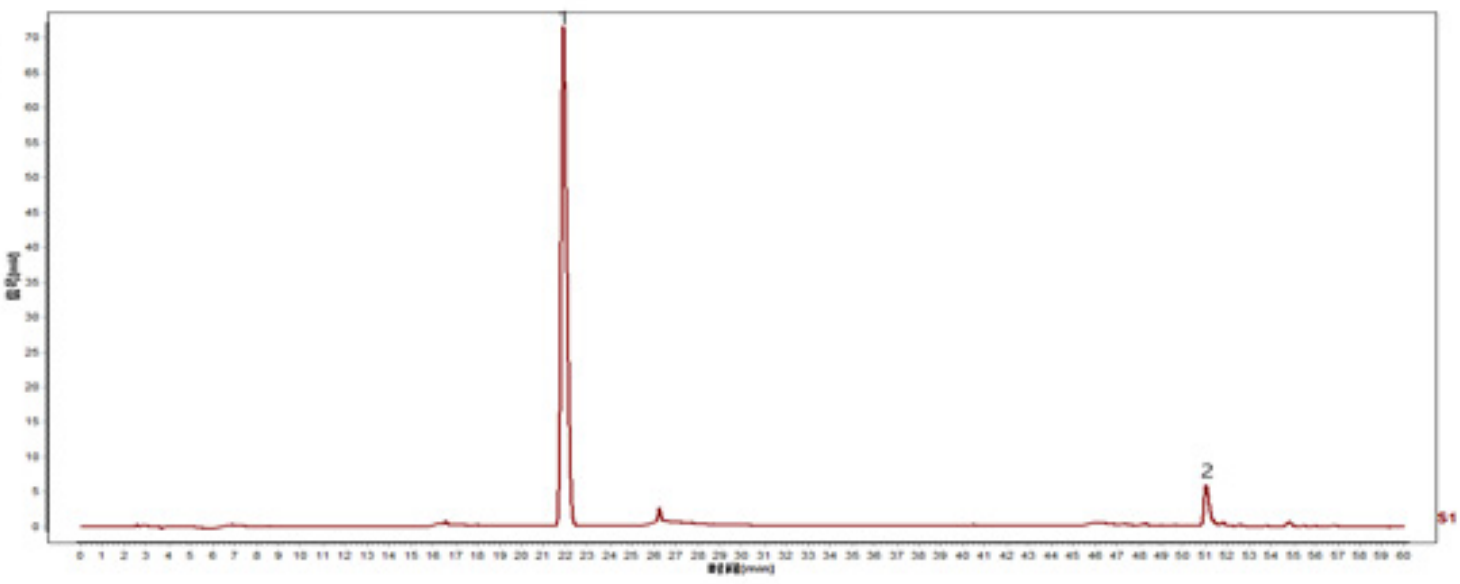

$\mathrm{D}$

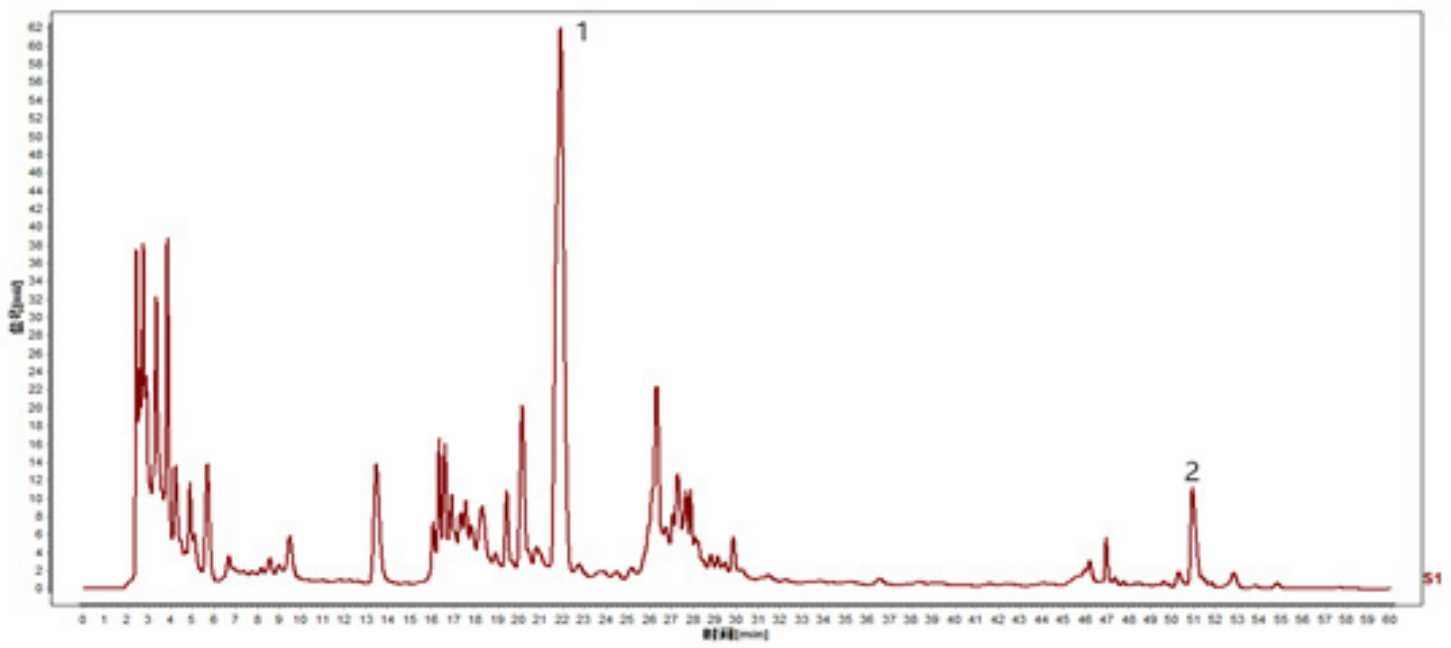

Figure 2: Preparation of different treatments.

(A). S. boulardii under the microscope.

(B). The macroscopic view of Dangshen polysaccharide.

(C). HPLC chromatogram of glycyrrhizin acid and liquidity in the reference solution.

(D). HPLC chromatogram of glycyrrhizin acid and liquidity in Sijunzi decoction. Peak 1: liquidity, Peak 2: glycyrrhizin acid.

\section{Different treatments alleviate DSS-induced colitis in} mice

An outline of the animal experiments and treatment design is shown in Figure 1. Before induction of colitis, four different treatments were administered orally for three weeks. Body weight
B

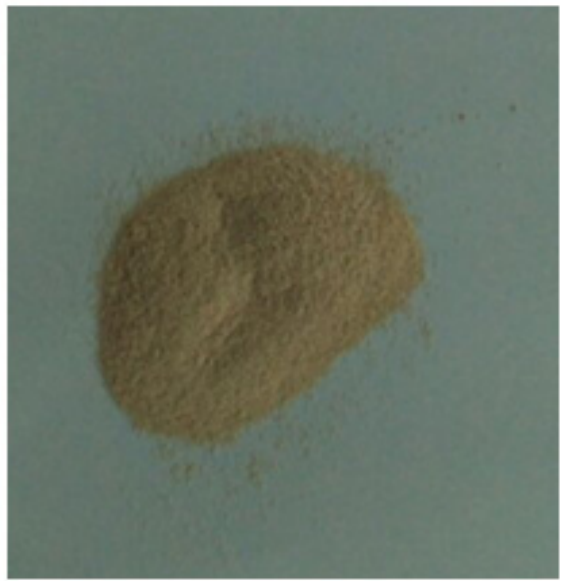



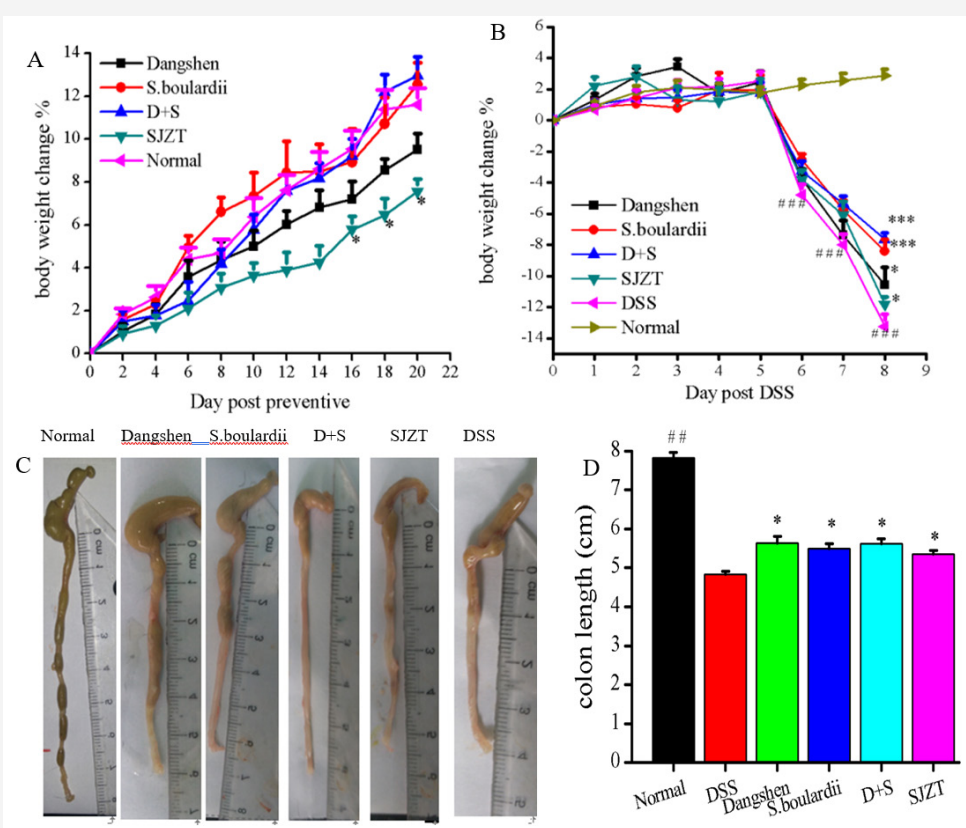

Figure 3: Different treatments alleviate DSS-induced colitis in mice.

(A). Daily body weight increase (relative to controls) before DSS administration, ${ }^{*} \mathrm{P}<0.05$.

(B-C). Daily body weight loss post-colitis treatment. ${ }^{*} P<0.05$, ${ }^{* * *} P<0.001$ vs DSS, \#\#\#P<0.001 vs controls

(D). Colon length after each treatment relative to DSS administration, ${ }^{*} P<0.05$, \#\#P<0.01. Differences were assessed by ANOVA ( $\left.n=6 / g r o u p\right)$.

After induction of colitis (2.5\% DSS solution for 7 days) the four treatments were continued administered until the end of study. All treatments significantly inhibited weight loss by day 30 relative to the DSS-only group $(\mathrm{P}<0.05)$, especially $\mathrm{S}$. boulardii and $\mathrm{D}+\mathrm{S}$ significantly $(\mathrm{P}<0.001)$ (Figure $3 \mathrm{~B})$. Total colon length decreased in all DSS-treated mice relative to controls, while treatment groups A-D showed a slight increase in colon length relative to the DSSonly group $(\mathrm{P}<0.05$, Figure $3 \mathrm{C} \& 3 \mathrm{D})$.

The degree of colonic inflammation was further confirmed by histological analysis. While control mice had intact surface epithelia, stroma, cryptal glands, and submucosae, DSS-treated mice showed surface epithelium damage, cryptal gland disruption and infiltration of lymphocytes.

All treatment groups had significantly lower histology scores than DSS-treated mice $(\mathrm{P}<0.05)$. In the $\mathrm{S}$. boulardii and Dangshen $+S$. boulardii groups, surface epithelium and cryptal glands were more intact than in the Dangshen and SJZT groups (Figure 4A). There was no obvious infiltration of inflammatory cells in any of the treated groups, especially not in the D+S group (Figure 4B).
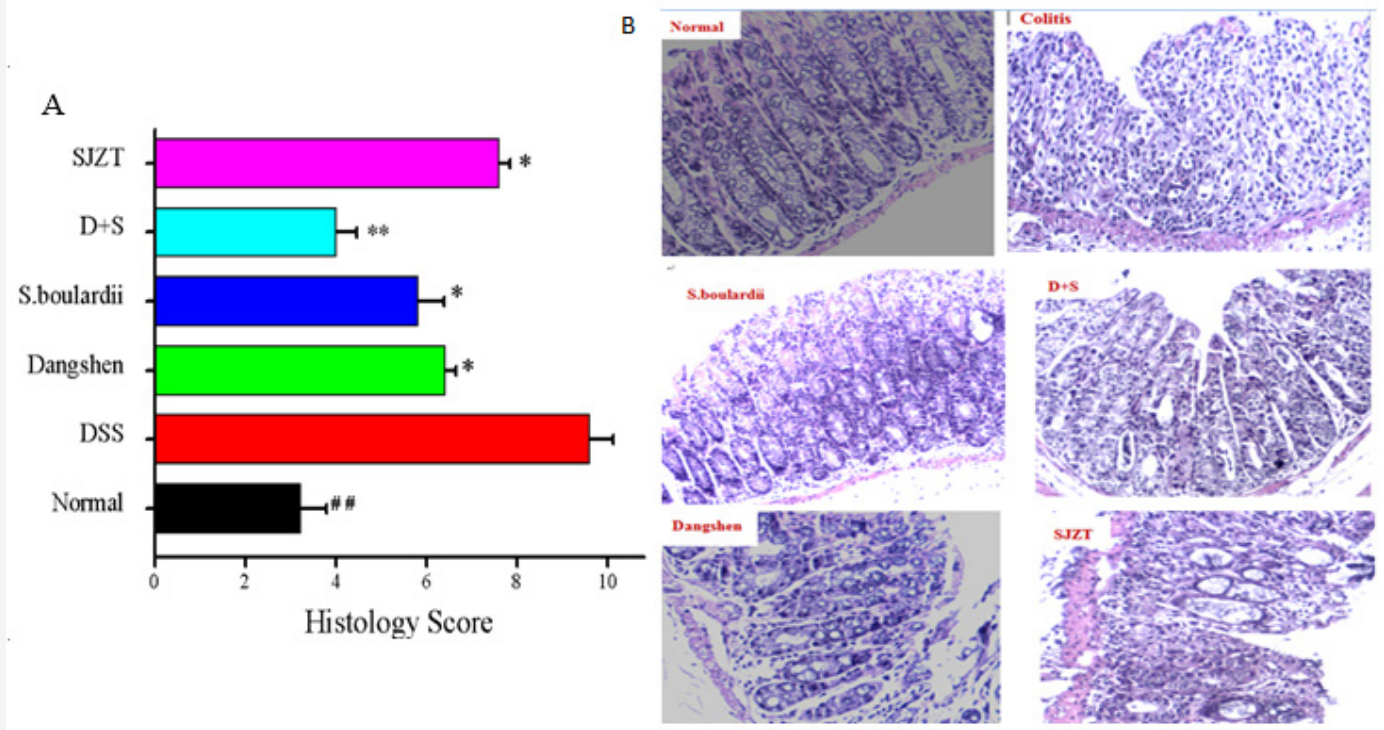

Figure 4: Different treatments attenuated the pathology associated with DSS-induced colitis.

(A). Histological data summary.

(B). Representative histological images (magnification 200x) are shown. Differences were assessed by ANOVA, ${ }^{*} P<0.05,{ }^{* *} P<0.01$, \# \#P< 0.01 . 


\section{Gut microbiome structure in response to different treatments in DSS-induced colitis}

Sequencing the V3-V4 16S hypervariable region produced 2387053 high quality sequences from fecal samples collected on days 1, 21 and 30, with a mean of $43400 \pm 1901$ sequences per sample. The mean number of OTUs per sample was 373 . Rarefaction and diversity analysis show that the majority of the gut microbial diversity was captured at the current sequencing depth, with few new OTUs anticipated at increased sequencing depths (Figure 5A).

All treatments increased gut microbiome richness in mice by day 21 relative to baseline levels at day 1 (Figure 5A), especially
$\mathrm{D}+\mathrm{S}(\mathrm{P}<0.01)$, Dangshen and S. boulardii $(\mathrm{P}<0.05)$, and to a lesser extent SJZT ( $>0.05$, Figure 5B). Post-treatment (day 30), all groups had slightly more unique OTUs than DSS-only mice, but this was not significant (Figure 5C).

In terms of diversity, the two polysaccharide-rich groups (Dangshen, D+S) had significantly higher Shannon indices than control mice before colitis (Figure 5E $\mathrm{P}<0.05$ ), while the SJZT group appeared unchanged. Acute colitis rapidly decreased diversity however, the four treatments all significantly increased diversity (Figure 5F, $\mathrm{P}<0.05$ ).
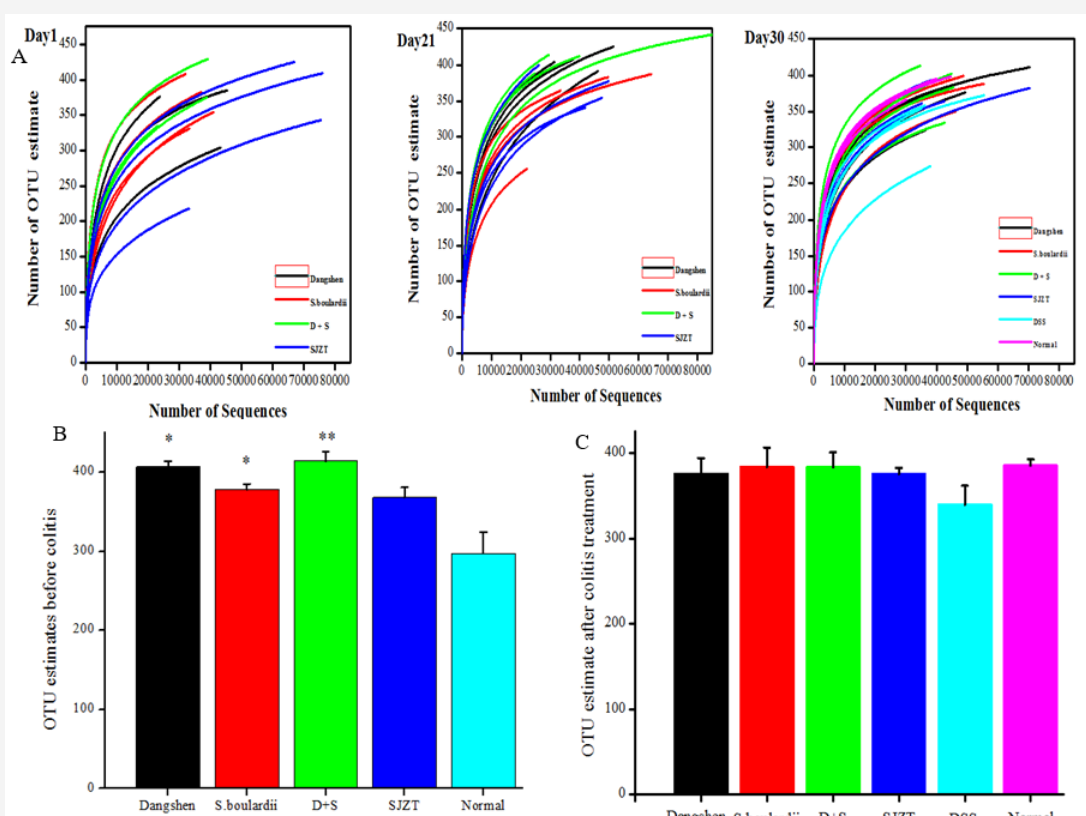

her of Sequences
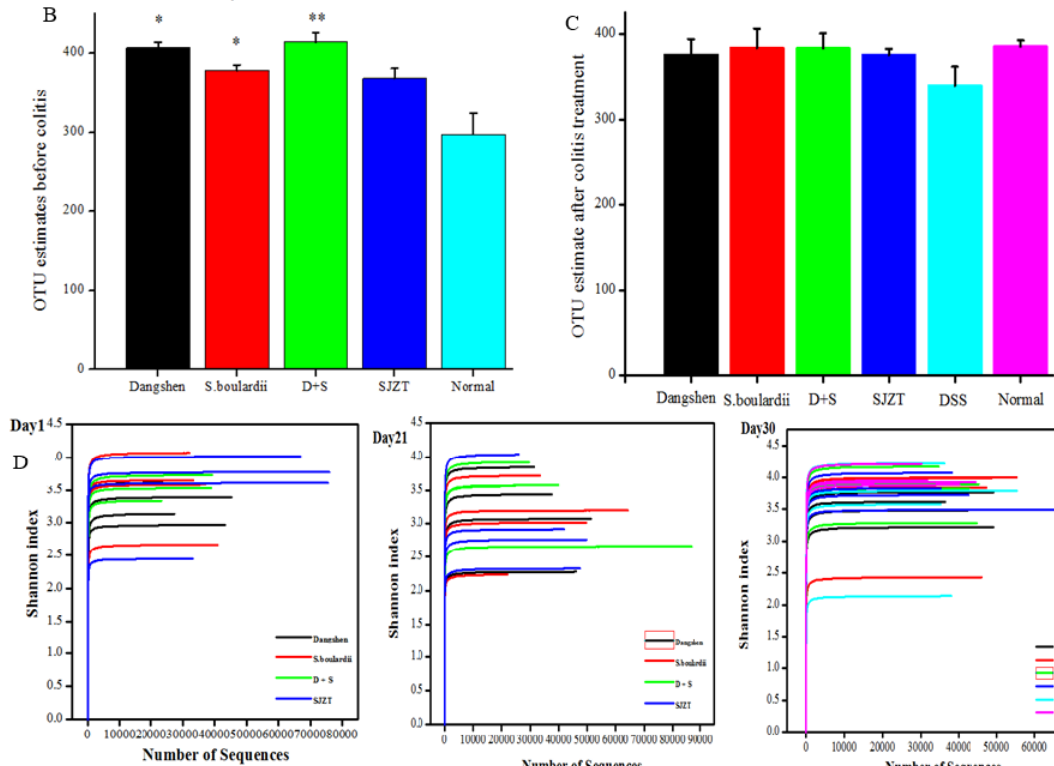

Number of Sequences

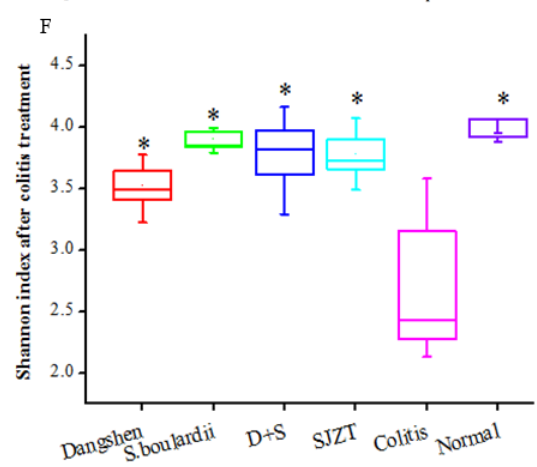

Figure 5: Diversity and richness of the gut microbiome in different treatments groups.

(A). Rarefaction curves on days 1, 21 and 30 , respectively.

(B-C). OTU estimates of each group on day 21 and day 30 , respectively $\left({ }^{*} \mathrm{P}<0.05\right.$ vs normal, where normal values represent the data from all mice at day 1).

(D). Shannon indices on days 1,21 and 30 , respectively.

(E-F). Shannon index of each group before colitis ( ${ }^{*} \mathrm{P}<0.05$ vs controls) and after colitis ( ${ }^{*} \mathrm{P}<0.05$ vs $\left.\mathrm{DSS}\right)$. 
The overall gut microbiome structure in response to the various treatments were further analyzed by uni Frac distance-based PCoA, which revealed that control mice had distinct microbiomes compared to all the other groups. All four treatments, except for a few outlier samples, modulated the colitis-associated microbiome, and the different treatments tended to form separate clusters (Figure 6A). NMDS analysis (Bray-Curtis distance) supported the
PCoA-based result and further showed that bacterial communities in the Dangshen group are more homogenous than other groups, followed by the SJZT group (Figure 6B). These findings were confirmed by hierarchical clustering analysis and suggest that the four treatments alter the microbiome in the inflamed colon (Figure 6C).
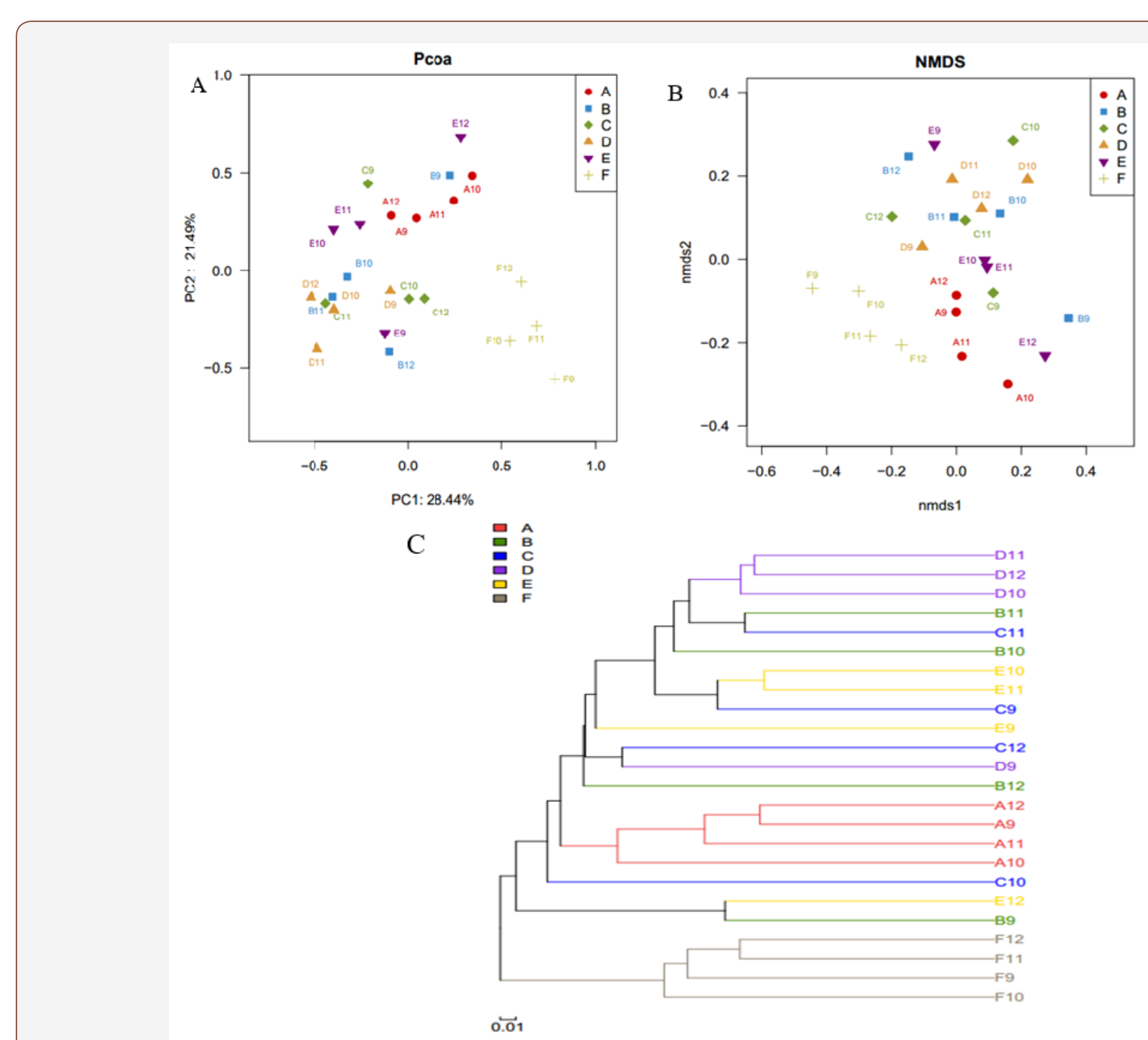

nmds1

Figure 6: The gut microbiome structure changed significantly following induction of colitis.

(A). PCoA plot based on Unirac distances.

(B). NMDS plot based on Bray-Curtis distances.

(C). Dendrogram (hierarchical clustering) of all samples.

\section{Gut microbiome composition in response to different} treatments in DSS-induced colitis

Atbaseline (day 1), Firmicutes, Bacteroidetes and Proteobacteria were the three, Firmicutes, Bacteroidetes and Proteobacteria are the three most abundant phyla, with Firmicutes and Bacteroidetes accounting for about $91 \%$ of reads. Following treatment, both Dangshen (74.97\% vs $79.35 \%)$ and Sijunzi (75.43\% vs $79.82 \%)$ groups had increased proportions of Bacteroidetes. Meanwhile, Bacteroidetes were decreased in the S. boulardii $(81.98 \%$ vs
$65.48 \%)$ and D+S (75.48\% vs 70.97\%) groups. Firmicutes were less abundant in the Dangshen (13.10\% vs $12.88 \%)$ and SJZT groups (16.86\% vs $10.64 \%)$, while the S. boulardii ( $11.70 \%$ vs $16.37 \%$ ) and D+S (12.62\% vs $16.52 \%$ ) groups had an increased proportion of Firmicutes. Proteobacteria was only decreased in the Dangshen group $(9.03 \%$ vs $6.82 \%)$, and increased in all three other groups, especially in the S. boulardii group, where it was increased more than three-fold $(4.62 \%$ vs $17.14 \%, \mathrm{P}<0.05$. Verrucomicrobia was significantly decreased $(\mathrm{P}<0.01)$ in all treatment groups (Figure 7A). 

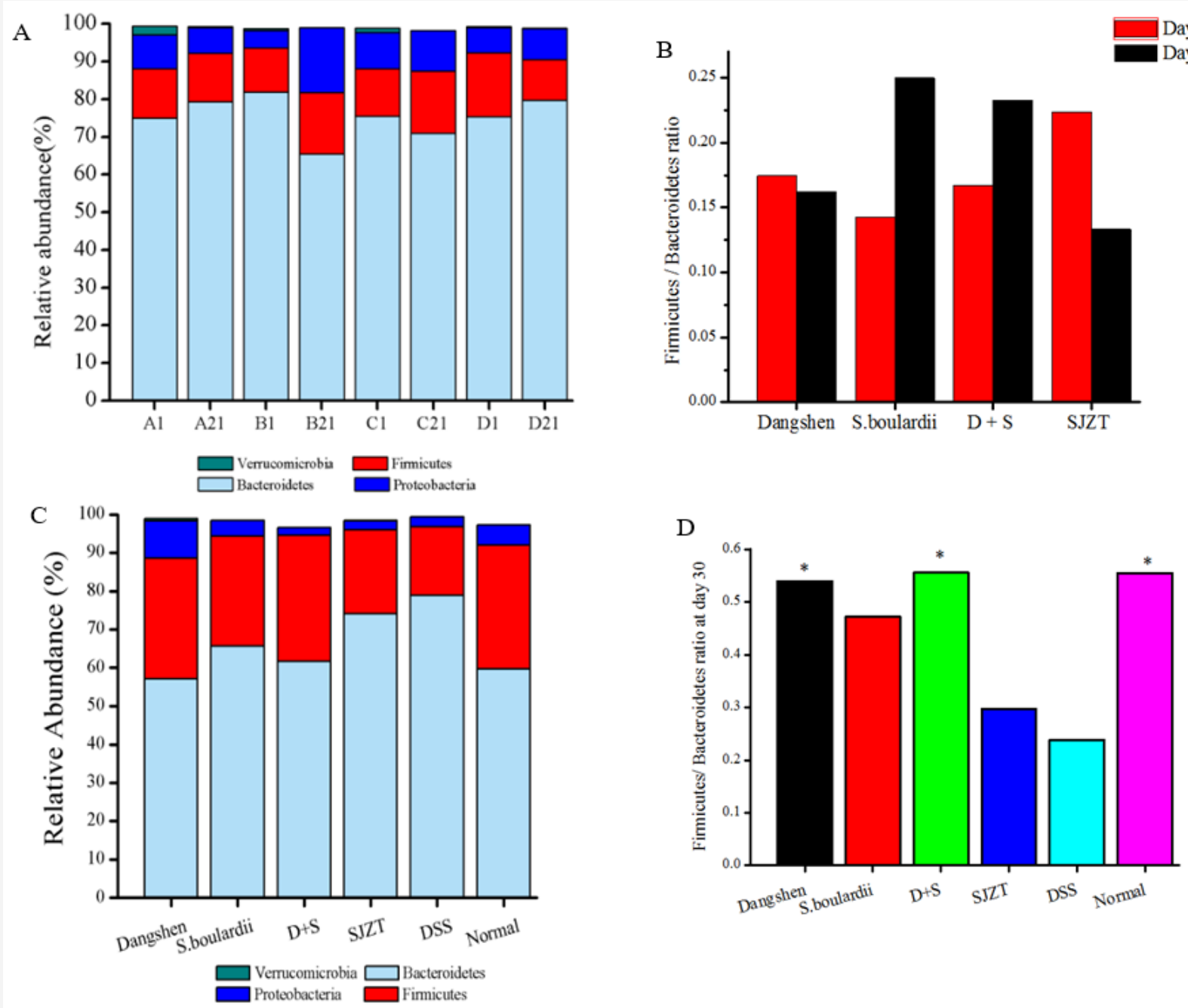

Figure 7: Treatment effect on microbial composition at phylum level.

(A). phylum-level composition pre- and post-preventative treatment (days 1 and 21).

(B). Firmicutes to Bacteroidetes ratio (F/B) post-preventative treatment.

(C). phylum-level composition post colitis treatments.at day 30 .

(D). F/B ratios post colitis treatments at day 30. Differences were assessed by Mann-Whitney test, relative to the DSS-only group * $P<0.05$.

DSS administration led to a remarkable decrease in Firmicutes $(17.77 \%$ vs $2.36 \%, \mathrm{P}<0.05)$ and Proteobacteria $(5.18 \%$ vs $2.52 \%$, $\mathrm{P}<0.05)$, with a significant increase in Bacteroidetes $(59.65 \%$ vs $78.98 \%, \mathrm{P}<0.05)$ relative to controls. These changes could however be mitigated by the treatments, especially by Dangshen polysaccharide which increased the proportion of Firmicutes (group A: $31.54 \%$, group C: $32.90 \%$ vs DSS-only: $17.77 \%, \mathrm{P}<0.05$ ) and decreased the proportion of Bacteroidetes (group A: 57.11\%, group C: $61.66 \%$ vs DSS-only: $78.98 \% \mathrm{P}<0.05$ ), Figure 7B. Relative to the DSS-only group, the Dangshen group had a significantly higher proportion of Proteobacteria $(9.65 \%$ vs $2.52 \%, \mathrm{P}<0.05)$, while Verrucomicrobia was slightly increased by Dangshen and SJZT (0.54\%, 0.047\% vs 0, $\mathrm{P}<0.05)$.

The Firmicutes to Bacteroidetes ratio $(\mathrm{F} / \mathrm{B})$ is used as an indicator of the gut microbial composition and was used to evaluate colitis remission and relapse. Post colitis treatment, F/B ratios decreased in the Dangshen and SJZT groups and increased in the S. boulardii and D+S groups (Figure 7C). Induction of colitis
(DSS-only)significantly decreased the F/B ratio relative to Normal mice $(0.55$ vs $0.24, \mathrm{P}<0.05)$, while all four preventative treatments increased the $\mathrm{F} / \mathrm{B}$ ratio (Figure $7 \mathrm{D}$ ), which was significant in the Dangshen and $\mathrm{D}+\mathrm{S}$ groups $(\mathrm{P}<0.05)$, where $\mathrm{F} / \mathrm{B}$ ratios were close to that of controls $(0.57,0.56$ vs 0.55 , respectively).

We next compared community differences at family level (Figure 8A \& 8B), which again highlighted the modulating effect of preventative treatment on gut microbial composition. Relative to day 1, all four treatments decreased the proportion of S24-7, especially Dangshen $(53.27 \%$ vs $30.52 \%, \mathrm{P}<0.05)$ and S. boulardii (44.37\% vs $23.50 \%, \mathrm{P}<0.05)$. Prevotellaceae was increased in all four treatment groups, and significantly so in the Dangshen $(16.27 \%$ vs $45.67 \%, \mathrm{P}<0.05)$ and $\mathrm{D}+\mathrm{S}(18.05 \%$ vs $42.84 \%, \mathrm{P}<0.05)$ groups. S. boulardii promoted Heliobacteria growth $(3.54 \%$ vs $14.87 \%$, $\mathrm{P}<0.05)$; SJZT decreased Rikenellaceae $(5.37 \%$ vs $1.39 \%, \mathrm{P}<0.05)$, Porphyromonadaceae $(1.52 \%$ vs $0.72, \mathrm{P}<0.05)$ and Bacteroide $(1.92 \%$ vs $0.84 \%, \mathrm{P}<0.05)$; and $\mathrm{D}+\mathrm{S}$ decreased Verrucomicrobiaceae (1.16\% vs $0.001 \%, \mathrm{P}<0.05)$. 

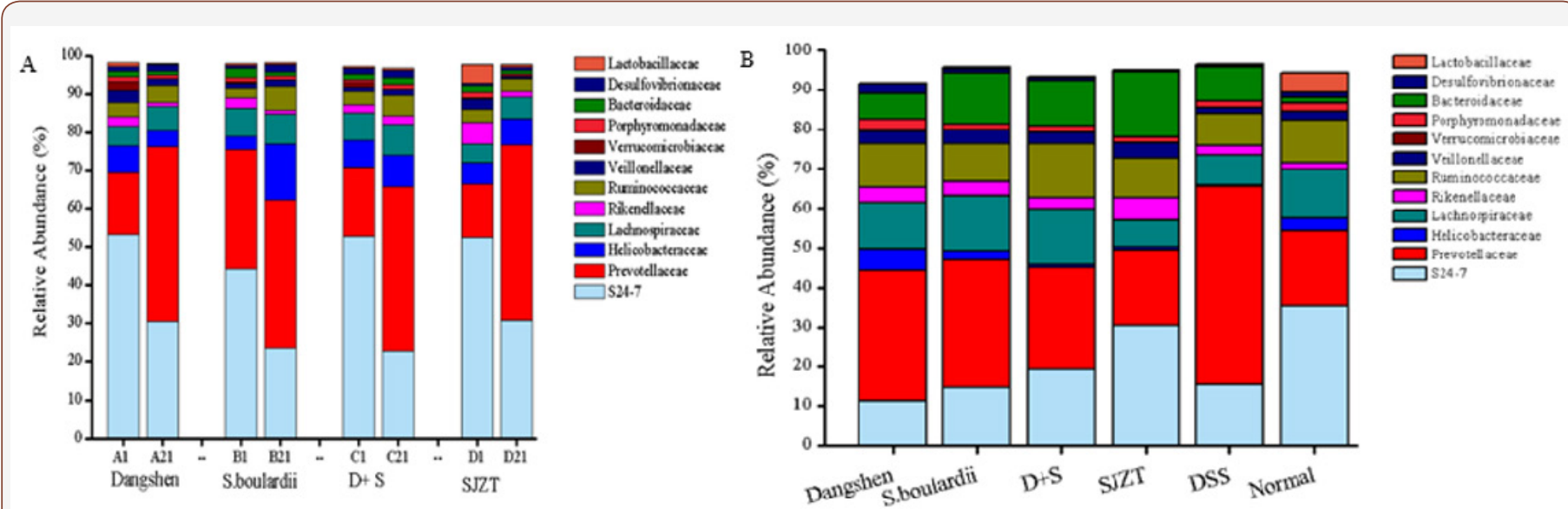

Figure 8: Treatment effect on microbial composition at family level.

(A). family-level composition pre- and post-preventative treatment (days 1 and 21).

(B). family-level composition post colitis treatments.

Compared with control mice, DSS-induced colitis led to a drastic decrease in S24-7 (35.07\% vs 15.18\%, P<0.05), Lactobacillaceae $(4.98 \%$ vs $0.21 \%, \mathrm{P}<0.05)$ and Helicobacteraceae $(3.17 \%$ vs $0.27 \%, \mathrm{P}<0.05)$, while Prevotellaceae $(19.27 \%$ vs $50.37 \%, \mathrm{P}<0.05)$ and Bacteroidaceae $(1.34 \%$ vs $8.67 \%, \mathrm{P}<0.05)$ were obviously increased. In the treatment groups, S24-7 recovered in the SJZT group compared vs DSS-only $(15.18 \%$ vs $30.25 \%, \mathrm{P}<0.05)$;
Prevotellaceae was inhibited by all four treatments, especially by Dangshen and SJZT $(\mathrm{P}<0.05)$, with Prevotellaceae levels returning to baseline in the SJZT group. Verrucomicrobiaceae was restored in the Dangshen and SJZT groups, while Dangshen and S.boulardii increased Helicobacteraceae abundance compared vs DSS-only $(5.23 \%, 2.22 \%$ vs $0, \mathrm{P}<0.05)$.

\section{Specific treatment-associated taxa in colitis mice}

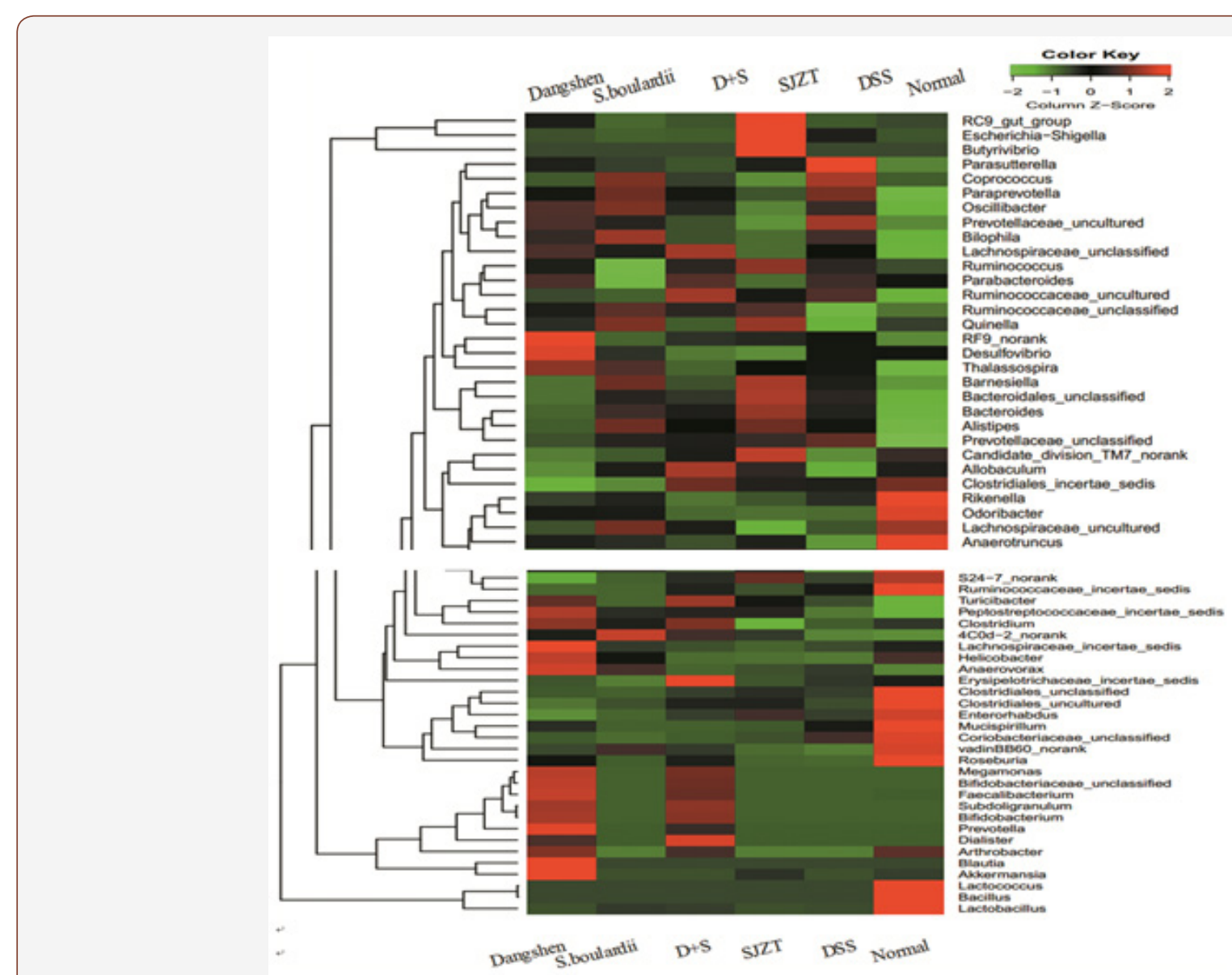

Figure 9: Specific treatment-associated taxa in colitis mice. Heatmap of fecal microbiota at genus level of top 60 most abundant genera among the six group. 
To further compare treatment-specific changes to gut microbial the top 60 most abundant genera (omitting a few rarely reported genera) were selected for comparison between groups (Figure 9). Mice with DSS-induced acute colitis (Group E) showed signs of dysbiosis, marked by a significant decrease in beneficial bacteria and an increase in pathogenic bacteria. Lactobacillus $(5.07 \%$ vs $0.17 \%, \mathrm{P}<0.05)$, Bacillus $(0.38 \%$ vs $0, \mathrm{P}<0.05)$ and Lactococcus $(0.32 \%$ vs $0, \mathrm{P}<0.05)$ were all decreased in DSS-only vs control mice, while potentially harmful IBD-associated bacteria including Bacteroides $(1.37 \%$ vs $8.97 \%, \mathrm{P}<0.05)$, Paraprevotella $(0.16 \%$ vs $1.78 \%, \mathrm{P}<0.05)$, Escherichia Shigella $(0.03 \%$ vs $0.13 \%, \mathrm{P}<0.05)$, and Alistipes $(0.67 \%$ vs $2.40 \%, \mathrm{P}<0.05)$ were all notably increased in DSS-only treated mice.
Preventative treatment could however alter colitis-associated dysbiosis. Lactobacillus, a commonly used probiotic, was significantly increased in the two S. boulardii-containing groups $(\mathrm{P}<0.05)$ yet was only slightly increased in the Dangshen and SJZT groups relative to the DSS-only group, but still far from the levels in the normal group. Bifidobacteriaceae, unclassified, Bifidobacterium and a new probiotic, Arthrobacter, were all significantly increased in the Dangshen group, and increased (without significance) in the $\mathrm{D}+\mathrm{S}$ group. Bacillus and Lactococcus could not however be restored in any of the preventative treatment groups. Akkermansia - a new beneficial microbe that plays a key role in combating metabolic disorders [15] - was significantly increased in SJZT and Dangshen groups (Table 1).

Table 1: The relative abundance of representative beneficial bacterial genera in different groups. Difference were assessed by Kruskal-Wallis test followed by the Mann-Whitney U test.

\begin{tabular}{|c|c|c|c|c|c|c|c|c|c|c|c|c|}
\hline \multirow{2}{*}{$\begin{array}{c}\text { Taxonomy } \\
\text { Genus name }\end{array}$} & \multicolumn{6}{|c|}{ Relative Abundance (\%) } & \multirow{2}{*}{$\begin{array}{c}\text { Kruskal Wallis } \\
\text { test } \\
\end{array}$} & \multicolumn{5}{|c|}{$P$ value Mann-Whitney U test } \\
\hline & F\% & E\% & A\% & B\% & $\mathrm{C} \%$ & D\% & & F vs E & A vs E & B vs E & C vs E & D vs E \\
\hline Arthrobacter & 0.04 & 0 & 0.04 & 0 & 0.03 & 0 & 0.002 & 0.018 & 0.018 & 1 & 0.018 & 1 \\
\hline Akkermansia & 0.03 & 0 & 0.49 & 0 & 0 & 0.05 & 0.01 & 0.014 & 0.014 & 1 & 0 & 0.014 \\
\hline Bacillus & 0.38 & 0 & 0 & 0 & 0 & 0 & 0.006 & 1 & 1 & 1 & 1 & 1 \\
\hline Bifidobacterium & 0 & 0 & 0.1 & 0 & 0.09 & 0 & 0.007 & 1 & 0.014 & 1 & 0.014 & 1 \\
\hline Bifidobacteriaceae unclassified & 0 & 0 & 0.1 & 0 & 0.07 & 0 & 0.007 & 1 & 0.014 & 1 & 0.014 & 1 \\
\hline Lactobacillus & 5.07 & 0 & 0.05 & 0.45 & 0.37 & 0.07 & 0.005 & 0.02 & 0.081 & 0.02 & 0.02 & 0.042 \\
\hline Lactococcus & 0.32 & 0 & 0 & 0 & 0 & 0 & 0.006 & 0.02 & 1 & 1 & 1 & 1 \\
\hline Total & 5.84 & 0 & 0.78 & 0.45 & 0.56 & 0.12 & - & - & - & - & - & - \\
\hline
\end{tabular}

Group differences in potentially harmful bacteria are summarized (Table 2). All preventative treatments led to a decrease in the relative abundance of Paraprevotella, Parasutterella, and Prevotellaceae, uncultured; Bacteroides was significantly decreased only in the Dangshen group $(\mathrm{P}<0.05)$; Escherichia_Shigella was significantly decreased in all treatment groups except SJZT $(\mathrm{P}<0.05)$; Desulfovibrio was decreased in the S. boulardii and SJZT groups.

Table 2: The relative abundance of representative harmful bacterial genera in different groups. Difference were assessed by Kruskal-Wallis test followed by the Mann-Whitney $U$ test.

\begin{tabular}{|c|c|c|c|c|c|c|c|c|c|c|c|c|}
\hline \multirow{2}{*}{$\begin{array}{c}\text { Taxonomy } \\
\text { Genus name }\end{array}$} & \multicolumn{6}{|c|}{ Relative Abundance (\%) } & \multirow{2}{*}{$\begin{array}{c}\text { Kruskal Wallis } \\
\text { test } \\
\end{array}$} & \multicolumn{5}{|c|}{$P$ value Mann-Whitney $U$ test } \\
\hline & F\% & E\% & A\% & B\% & $\mathrm{C} \%$ & D\% & & F vs E & A vs E & B vs E & C vs E & D vs $\mathrm{E}$ \\
\hline Alistipes & 0.67 & 2.4 & 1.71 & 3.2 & 2.4 & 3.2 & 0.059 & 0.043 & 0.386 & 0.564 & 0.77 & 0.386 \\
\hline Bilophila & 0.02 & 0.03 & 0.03 & 0 & 0 & 0 & 0.534 & 0.776 & 0.554 & 0.384 & 0.77 & 1 \\
\hline Bacteroides & 1.37 & 8.97 & 6.12 & 13 & 12 & 16 & 0.022 & 0.02 & 0.56 & 0.77 & 0.56 & 0.08 \\
\hline Coprococcus & 0.04 & 0.11 & 0.04 & 0.1 & 0.1 & 0 & 0.154 & 0.245 & 0.245 & 0.773 & 0.25 & 0.11 \\
\hline Desulfovibrio & 1.21 & 1.19 & 2.27 & 1 & 0.6 & 0.4 & 0.069 & 0.386 & 0.248 & 0.773 & 0.89 & 0.386 \\
\hline Escherichia Shigella & 0.03 & 0.13 & 0.03 & 0 & 0 & 0.3 & 0.014 & 0.021 & 0.019 & 0.021 & 0.02 & 1 \\
\hline Prevotellaceae uncultured & 19.44 & 37.7 & 34.3 & 31 & 24 & 18 & 0.377 & 0.248 & 0.564 & 0.564 & 0.56 & 0.248 \\
\hline Paraprevotella & 0.16 & 1.78 & 1.21 & 1.7 & 1.2 & 0.8 & 0.017 & 0.021 & 0.248 & 1 & 0.25 & 0.083 \\
\hline Parabacteroides & 1.06 & 1.18 & 1.26 & 0.5 & 1.3 & 0.8 & 0.557 & 0.773 & 0.773 & 0.083 & 0.66 & 0.248 \\
\hline Parasutterella & 0.41 & 1.49 & 0.76 & 0.6 & 0.6 & 0.7 & 0.227 & 0.083 & 0.083 & 0.083 & 0.08 & 0.149 \\
\hline Oscillibacter & 0.35 & 1.69 & 1.89 & 2 & 1.2 & 0.7 & 0.011 & 0.021 & 0.386 & 0.564 & 0.77 & 0.043 \\
\hline Total & 24.09 & 54.27 & 47.91 & 49.54 & 40.34 & 38.6 & - & - & - & - & - & - \\
\hline
\end{tabular}

Interestingly, there were significant increases in certain SCFAproducing bacteria, with 9 of the 13 identified genera significantly enriched in at least one treatment group relative to the DSS-only group (D) (Table 3); Butyrivibrio, Quinella, and Anaerotruncus were significantly enriched in the SJZT group $(\mathrm{P}<0.05)$. All baculum Blautia and Odoribacter were marginally increased in all treatment groups $(\mathrm{P}>0.05)$; Faecali bacterium, Mega monas, Prevotella,
Subdoligranulum and Dialister were increased in the Dangshen and $\mathrm{D}+\mathrm{S}$ groups, but not in the S. boulardii-only group, which suggests that Dangshen polysaccharide (and not S. boulardii) may be mitigating these effects; Roseburia was markedly increased in all treatment groups, especially in the Dangshen and S. boulardii groups, which also promoted Anaerovorax growth. 
Table 3: Group-specific relative abundances of SCFA-producing bacteria. Difference were assessed by Kruskal-Wallis test followed by the MannWhitney U test.

\begin{tabular}{|c|c|c|c|c|c|c|c|c|c|c|c|c|}
\hline \multirow{2}{*}{$\begin{array}{c}\text { Taxonomy } \\
\text { Genus name }\end{array}$} & \multicolumn{6}{|c|}{ Relative_Abundance (\%) } & \multirow{2}{*}{$\begin{array}{c}\text { Kruskal wallis } \\
\text { test }\end{array}$} & \multicolumn{5}{|c|}{ P value_Mann-Whitney $U$ test } \\
\hline & F\% & $\mathbf{E} \%$ & $\mathbf{A} \%$ & B\% & $\mathrm{C} \%$ & D\% & & F vs E & A vs $E$ & B vs E & C vs E & D vs E \\
\hline Anaerotruncus & 0.78 & 0.13 & 0.37 & 0.3 & 0.3 & 0.4 & 0.014 & 0.021 & 0.083 & 0.149 & 0.08 & 0.021 \\
\hline Allobaculum & 0.04 & 0.01 & 0.02 & 0 & 0.1 & 0 & 0.269 & 0.08 & 0.659 & 0.882 & 0.11 & 0.081 \\
\hline Anaerovorax & 0 & 0 & 0.05 & 0 & 0 & 0 & 0.001 & 1 & 0.019 & 0.036 & 1 & 0.096 \\
\hline Butyrivibrio & 0 & 0 & 0 & 0 & 0 & 0.1 & 0.006 & 1 & 1 & 1 & 1 & 0.047 \\
\hline Blautia & 0 & 0 & 0.02 & 0 & 0 & 0 & 0.416 & 1 & 0.307 & 1 & 1 & 1 \\
\hline Dialister & 0 & 0 & 0.02 & 0 & 0 & 0 & 0.001 & 1 & 0.014 & 1 & 0.01 & 1 \\
\hline Faecalibacterium & 0.03 & 0 & 1.36 & 0 & 1 & 0 & 0.002 & 1 & 0.014 & 1 & 0.01 & 1 \\
\hline Megamonas & 0 & 0 & 1.05 & 0 & 0.8 & 0 & 0.002 & 1 & 0.014 & 1 & 0.01 & 1 \\
\hline Odoribacter & 1.18 & 0.56 & 0.83 & 0.8 & 0.6 & 0.6 & 0.572 & 0.248 & 0.386 & 0.386 & 0.77 & 0.663 \\
\hline Prevotella & 0 & 0 & 2.67 & 0 & 1.3 & 0 & 0.001 & 1 & 0.014 & 1 & 0.01 & 1 \\
\hline Quinella & 2.31 & 1.43 & 2.53 & 3.6 & 2.1 & 3.8 & 0.547 & 0.773 & 0.564 & 0.248 & 0.25 & 0.043 \\
\hline Roseburia & 0.48 & 0 & 0.16 & 0 & 0.1 & 0 & 0.007 & 0.014 & 0.014 & 0.047 & 0.01 & 0.047 \\
\hline Subdoligranulum & 0 & 0 & 0.18 & 0 & 0.2 & 0 & 0.001 & 1 & 0.014 & 1 & 0.01 & 1 \\
\hline Total & 4.82 & 2.13 & 9.26 & 4.78 & 6.4 & 4.85 & - & - & - & - & - & - \\
\hline
\end{tabular}

\section{Discussion}

The human gut microbiome plays a vital role in maintaining body homeostasis. Changes in bacterial compositional (i.e. dysbiosis) can profoundly impact human health, which makes the microbiome an important therapeutic target. Both bacterial diversity and abundance is altered in human IBD; however, whether dysbiosis is a consequence or cause of IBD remains controversial. The success of manipulating the gut microbiome to cure or alleviate the symptoms of IBD has encouraged the use of probiotic and prebiotic therapies.

IBD-directed therapies however have variable success rates and a high risk of severe side effects with long-term use. Therefore, in recent years, the use of CAMs has become widespread due to their effectiveness and absence of side effects [16]. Besides probiotics and prebiotics, Chinese traditional medicines may be a viable microbiome-manipulating therapeutic option.

In this study, we clearly demonstrated the effectiveness of four different CAMs in relieving DSS-induced colitis. Although most Chinese herbal medicines and their active polysaccharides (e.g. Astragalus membranous, Rheum rhabarbarin polysaccharide) have anti-inflammatory effects in a mice colitis model [17], we are the first to report the use of Dangshen [Codonopsis pilosula (Franch.) Nannf] polysaccharide in the treatment of colitis in mice. In fact, Dangsheng polysaccharide was even more effective than the herbal medicine SJZT, which confirms that Dangshen polysaccharide is the key active ingredient in SJZT. In addition, we found that S. boulardii significantly alleviates the symptoms of colitis, which is in agreement with previous research [18]. S. boulardii in combination with Dangshen polysaccharide, was more effective than S. boulardii alone, which suggests a possible synergistic or symbiotic effect.

Following 21 days of preventive treatments (before induction of colitis), a high proportion of Bacteroidetes were present in the Dangshen and SJZT groups. Besides Dangshen polysaccharide, the herbal decoction also contains other plant polysaccharides. On the other hand, Bacteroidetes are well equipped for carbohydrate metabolism [19] so it is not surprising that Bacteroidetes are increased in these groups. Enrichment with Bacteroidetes in polysaccharide-treated mice could be related to polysaccharide degradation in the gut. Furthermore, mice body weight was increased in the two S. boulardii-containing groups compared with the Dangshen and SJZT groups. This was associated with increased $\mathrm{F} / \mathrm{B}$ ratios in $\mathrm{S}$. boulardii-containing groups, which has been associated with enhanced energy harvesting [20].

The $\mathrm{F} / \mathrm{B}$ ratio is also a key index in evaluating IBD remission and relapse [20,31]. All treatments inhibited the DSS-induced decrease in the F/B ratio, with significantly increased $\mathrm{F} / \mathrm{B}$ ratios in the Dangshen and $D+S$ groups $(P<0.05)$ to values similar to that of the normal (non-DSS group). Modulating F/B ratios in DSS-induced colitis may represent an important underlying mechanism of these treatments.

Another interesting feature is that Proteobacteria was significantly increased, especially in the S. boulardii group after 21 days of treatment,yet increased only in the Dangsheng group postcolitis (day 30). Proteobacteria can induce a specific IgA response to regulate maturation of the immune maturation [22]. It has also been reported that $\mathrm{S}$. boulardii stimulates intestinal IgA production [23], and that total polysaccharide extracted from SJZT can restore IgA production in a cyclophosphamide-induced lymphoid tissue injury model [24]. Therefore, the ability of S. boulardii and Dangshen polysaccharide to induce IgA may promote the growth of Proteobacteria.

The most important characteristic of a good prebiotic is to selectively stimulate the growth of host-beneficial bacteria such as Bidifo bacteria and Lactobacilli. Bifidobacterium, Bifido bacteriaceae, unclassified and Lactobacillus were only seen in the Dangshen and D+S group.

Arthrobacter produces hydrolytic enzymes (endoinulinases [25]) that degrade inulin into common prebiotic fructo oligosaccharides. Arthrobacter arilaitensis can produce $\beta$-fructo 
furanosidase, which is used to synthesize the prebiotic kestose [26]. Most Arthrobacter species are probiotics and both Arthrobacter agilis and Arthrobacter citrus have been used to treat IBD [27]. In this study, Arthrobacter growth was facilitated by Dangshen polysaccharide, which supports its prebiotic value.

Bacterially-derived SCFAs are major products of prebiotic metabolism. We found that Anaerovorax, Roseburia, Prevotella, Megamonas, Dialister, Faecali bacterium and Subdoli granulum were remarkably increased in the Dangshen group. Apart from Anaerovorax and Roseburia, the other taxa were specifically increased in the Dangshen group.

Prevotella is associated with consumption of a diet rich in fiber because of its outstanding ability for cellulose and xylan hydrolysis. A long-term fiber-rich diet therefore promotes the growth of Prevotella, along with increased production of SCFAs [28]. Mega monas and Faecalis bacterium are known producers of SCFA, which are decreased in IBD patients [29]. F. prausnitzii, the representative species of the Faecali bacterium genus, is a commonly administered intestinal probiotic in recent years. It produces SCFAs and increases production of IL-10 and TGF- $\beta$ to control colonic inflammation by regulating the Th17/Treg balance. The common prebiotic inulin greatly facilitates Faecalibacterium growth [30]. In this study, when colitis mice were treated with Dangshen polysaccharide, Prevotella, Mega monas and Faecali bacterium all increased to > 1\% $2.67 \%$, $1.05 \%, 1.36 \%$ respectively), which supports the value of Dangshen polysaccharide as a powerful prebiotic.

Other SCFA-producing bacteria (Butyrivibrio, Quinella and Anaerotruncus) were seen only in the SJZT group, which suggests the presence of small active molecules or medicinal polysaccharides other than Dangshen polysaccharide that promote the growth of these beneficial microbes. The exact composition of SJZT however requires further study.

Roseburia, which produces SCFAs and is now used to treat UC [31], was increased in all four treatment groups. Odoribacter was reduced in DSS-induced colitis, and Odoribacter splanchnus, a typical representative of the genus Odoribacter, is a known producer of acetate and propionate [32]. S. boulardii mildly promoted Odoribacter growth. Anaerovorax metabolizes putrescine to acetate and butyrate [33] and Anaerovorax was remarkably increased in the S. boulardii group. Taken together, these results suggest that $\mathrm{S}$. boulardii is an effective probiotic that facilitates the growth of the SCFA-producing bacteria: Roseburia, Odoribacter and Anaerovorax.

Lactobacillus was also increased significantly in the S. boulardii group. Other yeasts have been reported to stimulate the growth of lactic acid strains in fermented products [34]. Our result therefore suggests that $\mathrm{S}$. boulardii also has the ability to promote Lactobacillus growth in vivo.

Akkermansia plays a key role in preventing obesity and metabolic disease. A. muciniphila is decreased significantly in diabetes patients. Recent research suggests that metformin may act by facilitating Akkermansia proliferation [35]. Akkermansia is also decreased in IBD patients and a previous study reported that extracellular vesicles derived from Akkermansia muciniphila protect against DSS-induced colitis [36]. In addition, administration of common oligofructose-containing prebiotics completely restored Akkermansia levels in both genetically and high fat-fed obese mice [15]. In this study, we found that Dangshen polysaccharide restored Akkermansia abundance in acute colitic mice, which suggests that Dangshen polysaccharide may be useful as a prebiotic in the prevention of obesity and diabetes.

Desulfovibrio is the most important member of the sulphatereducing bacteria. It breaks down SCFA and amino acids to produce $\mathrm{H} 2 \mathrm{~S}$ that harm intestinal epithelial cells. Desulfovibrio is increased in the inflamed colon [37], which can be counteracted by S. boulardii and SJZT. Bacteroides was increased in acute colitis, which is in agreement with a previous study [38]. However, only Dangshen polysaccharide was able to control this increase in Bacteroides, while S. boulardii even promoted its growth. This is in agreement with a previous report where Bacteroides was dramatically increased by S. boulardii administration in type 2 diabetic mice [10]. This may be due to the ability of Bacteroides to utilize t cell walls as, which is made up of $\beta$-glucans, as an energy source [8]. However, it remains unclear why SJZT increased the proportion of Bacteroides.

Prevotellaceae, uncultured and Parasutterella, which are enriched in IBD patients [17] and in colorectal cancer tissue [39], were inhibited by all four treatments at varying degrees. Escherichia-Shigella is a common pathogenic bacterium that was induced by DSS administration. All treatments, except SJZT, inhibited Escherichia-Shigella growth. Taken together, the common mechanism, shared by all four treatments, was to inhibit harmful bacterial growth.

\section{Conclusion}

Chinese traditional herbal medicine SJZT, Dangshen polysaccharide and S. boulardii were all effective in alleviating DSSinduced colitis. Promoting beneficial and inhibiting pathogenic bacteria may be the shared mechanism of these potential CAM drugs in improving colonic health.

\section{Data Availability}

The data used to support the findings of this study are available from the corresponding author upon request.

\section{Funding Statement}

This study was funded by Jiangsu Science and Technology Major Project (No. BA2016036), and Gansu Science and Technology Major Project (No. 17ZD2FA009).

\section{Conflicts of Interest}

The authors declare that there is no conflict of interest regarding the publication of this paper.

\section{References}

1. Kaplan GG (2015) The global burden of IBD: from 2015 to 2025. Nat Rev Gastroenterol Hepatol 12(12): 720-727.

2. Li J, Butcher J, Mack D, Stintzi A (2015) Functional Impacts of the Intestinal Microbiome in the Pathogenesis of Inflammatory Bowel Disease. Inflamm Bowel Dis 21(1): 139-153. 
3. Xu J, Lian F, Zhao L, Zhao Y, Chen X, et al. (2015) Structural modulation of gut microbiota during alleviation of type 2 diabetes with a Chinese herbal formula. ISME J 9(3): 552-562.

4. Yongxu S, Jicheng L (2008) Structural characterization of a water-soluble polysaccharide from the roots of Codonopsis pilosula and its immunity activity. Int J Biol Macromol 43(3): 279-282.

5. Xu X, Zhang X (2015) Lentinula edodes-derived polysaccharide alters the spatial structure of gut microbiota in mice. PLoS One 10(1): e0115037.

6. Chang CJ, Lin CS, Lu CC, Martel J, Ko YF, et al. (2015) Ganoderma lucidum reduces obesity in mice by modulating the composition of the gut microbiota. Nat Commun 6: 7489.

7. Garrido Mesa J, Algieri F, Rodriguez Nogales A, Utrilla MP, Rodriguez Cabezas ME, et al. (2015) A new therapeutic association to manage relapsing experimental colitis: Doxycycline plus Saccharomyces boulardii. Pharmacol Res 97: 48-63.

8. Jawhara S, Poulain D (2007) Saccharomyces boulardii decreases inflammation and intestinal colonization by Candida albicans in a mouse model of chemically-induced colitis. Med Mycol 45(8): 691-700.

9. Foligne B, Dewulf J, Vandekerckove P, Pignede G, Pot B (2010) Probiotic yeasts: anti-inflammatory potential of various non-pathogenic strains in experimental colitis in mice. World J Gastroenterol 16(7): 2134-2145.

10. Everard A, Matamoros S, Geurts L, Delzenne NM, Cani PD (2014) Saccharomyces boulardii administration changes gut microbiota and reduces hepatic steatosis, low-grade inflammation, and fat mass in obese and type 2 diabetic db/db mice. MBio 5(3): e01011-e01014

11. Liu Y, Yang Js (2005) Determination of Liquiritigenin, liquiritin, isoliquiritigenin and isoliquiritin in extract of traditional Chinese medicine Sijunzi Decoction by high-performance liquid chromatography. JOURNAL OF CHINESE PHARMACEUTICAL SCIENCES 14: 227.

12. Egger B, Bajaj Elliott M, MacDonald TT, Inglin R, Eysselein VE, et al. (2000) Characterisation of acute murine dextran sodium sulphate colitis: cytokine profile and dose dependency. Digestion 62 (4): 240-248.

13. Schloss PD, Westcott SL, Ryabin T, Hall JR, Hartmann M, et al. (2009) Introducing mothur: open-source, platform-independent, communitysupported software for describing and comparing microbial communities. Appl Environ Microbiol 75(23): 7537-7541.

14. Fan W, Tang Y, Qu Y, Cao F, Huo G (2014) Infant formula supplemented with low protein and high carbohydrate alters the intestinal microbiota in neonatal SD rats. BMC microbiol 14: 279.

15. Everard A, Belzer C, Geurts L, Ouwerkerk JP, Druart C, et al. (2013) Cross-talk between Akkermansia muciniphila and intestinal epithelium controls diet-induced obesity. Proc Natl Acad Sci U S A 110(22): 90669071.

16. Langmead L, Rampton DS (2006) Review article: complementary and alternative therapies for inflammatory bowel disease. Aliment Pharmacol Ther 23(3): 341-349.

17. Ko JKS, Chik CW (2009) The protective action of radix Astragalus membranaceus against hapten-induced colitis through modulation of cytokines. Cytokine 47(2): 85-90.

18. Hu J, Franzen O, Pei Z, Itzkowitz S, Peter I (2013) P-237 Multiple Doublebarcoding $16 \mathrm{~S}$ Sequencing on the MiSeq Platform to Study the Gut Microbiome in Ashkenazi Jews with Crohn's Disease. Inflammatory Bowel Diseases 19: S119-S121.

19. Bolam DN, Sonnenburg JL (2011) Mechanistic insight into polysaccharide use within the intestinal microbiota. Gut microbes 2(2): 86-90.

20. Mariat D, Firmesse O, Levenez F, Guimaraes V, Sokol H, et al. (2009) The Firmicutes/Bacteroidetes ratio of the human microbiota changes with age. BMC Microbiol 9(9): 123.

21. Sokol H, Seksik P, Furet JP, Firmesse O, Nion Larmurier I, et al. (2009) Low counts of Faecalibacterium prausnitzii in colitis microbiota. Inflamm Bowel Dis 15(8): 1183-1189.
22. Mirpuri J, Raetz M, Sturge CR, Wilhelm CL, Benson A, et al. (2014) Proteobacteria-specific IgA regulates maturation of the intestinal microbiota. Gut Microbes 5(1): 28-39.

23. Rodrigues AC, Cara DC, Fretez SH, Cunha FQ, Vieira EC, et al. (2000) Saccharomyces boulardii stimulates sIgA production and the phagocytic system of gnotobiotic mice. J Appl Microbiol 89(3): 404-414.

24. Liu L, Zhou H, Wang PX, Hu Y (2000) Influence of total polysaccharide extracted from Sijunzi decoction on the intestinal mucosa associated lymphoid tissues of mice. Chin J Immunol 17: 204-206.

25. Singh RS, Singh RP (2010) Production of fructooligosaccharides from inulin by endoinulinases and their prebiotic potential. Food Technology and Biotechnology 48: 435-450.

26. Chu J, Wu X, Wu B, Wang R, He B (2014) Characteristics of an organic solvent-tolerant $\beta$-fructofuranosidase from Arthrobacter arilaitensis NJEM01 and efficient synthesis of prebiotic kestose. J Agric Food Chem 62(24): 5408-5411.

27. Trachtman IM (2013) Compositions and method for treatment and prophylaxis of inflammatory bowel disease. p. pp. Google Patents.

28. De Filippo C, Cavalieri D, Di Paola M, Ramazzotti M, Poullet JB, et al. (2010) Impact of diet in shaping gut microbiota revealed by a comparative study in children from Europe and rural Africa. Proc Natl Acad Sci U S A 107(33): 14691-14696.

29. Chen L, Wang W, Zhou R, Ng SC, Li J, et al. (2014) Characteristics of fecal and mucosa-associated microbiota in Chinese patients with inflammatory bowel disease. Medicine (Baltimore) 93(8): e51

30. Ramirez Farias C, Slezak K, Fuller Z, Duncan A, Holtrop G, et al. (2009) Effect of inulin on the human gut microbiota: stimulation of Bifidobacterium adolescentis and Faecalibacterium prausnitzii. Br J Nutr 101(4): 541-550.

31. Machiels K, Joossens M, Sabino J, De Preter V, Arijs I, et al. (2013) A decrease of the butyrate-producing species Roseburia hominis and Faecalibacterium prausnitzii defines dysbiosis in patients with ulcerative colitis. Gut 63(8): 2013-304833

32. Morgan XC, Tickle TL, Sokol H, Gevers D, Devaney KL, et al. (2012) Dysfunction of the intestinal microbiome in inflammatory bowel disease and treatment. Genome Biol 13(9): R79.

33. Matthies C, Evers S, Ludwig W, Schink B (2000) Anaerovorax odorimutans gen. nov, sp nov, a putrescine-fermenting, strictly anaerobic bacterium. Int J Syst Evol Microbiol 50 Pt 4: 1591-1594.

34. Narvhus JA, Gadaga TH (2003) The role of interaction between yeasts and lactic acid bacteria in African fermented milks: a review. Int J Food Microbiol 86(1-2): 51-60.

35. Forslund K, Hildebrand F, Nielsen T, Falony G, Le Chatelier E, et al. (2015) Disentangling type 2 diabetes and metformin treatment signatures in the human gut microbiota. Nature 528(7581): 262-266.

36. Kang Cs, Ban M, Choi EJ, Moon HG, Jeon JS, et al. (2013) Extracellular vesicles derived from gut microbiota, especially Akkermansia muciniphila, protect the progression of dextran sulfate sodium-induced colitis. PLoS One 8(10): e76520.

37. Loubinoux J, Bronowicki JP, Pereira IA, Mougenel JL, Le Faou AE (2002) Sulfate-reducing bacteria in human feces and their association with inflammatory bowel diseases. FEMS Microbiol Ecol 40(2): 107-112.

38. Videla S, Vilaseca J, Antolin M, Garcia Lafuente A, Guarner F, et al. (2001) Dietary inulin improves distal colitis induced by dextran sodium sulfate in the rat. Am J Gastroenterol 96(5): 1486-1493.

39. Chen W, Liu F, Ling Z, Tong X, Xiang C (2012) Human intestinal lumen and mucosa-associated microbiota in patients with colorectal cancer. PLos One 7(6): e39743. 